\title{
DESAFÍOS PARA EL LIDERAZGO EN UNA UNIVERSIDAD PRIVADA EN UN CONTEXTO DE POBREZA: IMPACTO DE LA FALTA DE ACCESO A LA EDUCACIÓN SUPERIOR EN TEKAX, YUCATÁN, MÉXICO
}

\author{
CHALLENGES FOR LEADERSHIP IN A PRIVATE UNIVERSITY \\ IN A CONTEXT OF POVERTY: IMPACT OF LACK OF ACCESS \\ TO HIGHER EDUCATION IN TEKAX, YUCATAN MEXICO
}

Juan Carlos Ortiz Navarro', Edith Juliana Cisneros Chacón²

\begin{abstract}
Resumen
El presente artículo define el constructo de pobreza y vulnerabilidad, las razones por las cuales la pobreza y la vulnerabilidad se anidaron en latinoamérica y, desde luego, en México. Del mismo modo, la investigación evidencia los niveles de pobreza existentes en México en la actualidad, asi como sus causas, particularmente en el municipio de Tekax, estado de Yucatán. Posteriormente, se hace una revisión y análisis sobre diferentes casos de éxito en combate a la pobreza, desarrollo económico y social por medio de estrategias de desarrollo de capital humano, intelectual y social, en paises cuyas características socioeconómicas eran similares a las condiciones de vida en México y el estado de Yucatán. De esta manera, se busca comprender por qué otras economías (principalmente las del este asiático) han logrado tener grandes avances en materia de desarrollo económico y social, mientras que México, a pesar de su gran capacidad económica, no ha podido de alcanzar los mismos resultados en cuanto a reducción de pobreza y vulnerabilidad, desarrollo económico y social. Para finalizar, con conclusiones y recomendaciones sobre directrices complementarias que la sociedad yucateca podría implementar, para mejorar los niveles de bienestar de la población en Tekax.
\end{abstract}

Palabras clave

Desarrollo económico, desarrollo social, desarrollo de capital intelectual, desarrollo de capital humano, pobreza y vulnerabilidad en Tekax, Yucatán.

\section{Abstract}

The present research work, in the first instance, defines the construct poverty and vulnerability, the reasons why poverty and vulnerability were nested in Latin America and of course in Mexico. In the same way, the investigation evidences the levels of poverty existing in Mexico at present, as well as, its causes particularly in the State of Yucatan, to later make a revision and analysis on different cases of success in the fight against poverty, economic and social development through human, intellectual and social capital development strategies, in countries whose socioeconomic characteristics are similar to living conditions in Mexico and the State of Yucatan, in order to understand why these other economies (mainly the Eastern of Asia) have achieved great progress in terms of economic and social development, while Mexico, despite its great economic capacity, has not been able to achieve the same results in terms of poverty and vulnerability reduction, economic and social development refer. To conclude and recommend complementary guidelines that the Yucatecan society could implement, to improve the welfare levels of population in Tekax.

Keywords

Economic development, social development, intellectual capital development, human capital development, poverty and vulnerability in Tekax, Yucatan. 


\section{Introducción}

Se eligió el municipio de Tekax en el estado de Yucatán para llevar a cabo el presente trabajo de investigación el cual forma parte de un trabajo doctoral en curso por varios motivos: 1) cuenta con 2 instituciones de educación superior: una pública y una privada); 2) el estudio de la tesis se centra en el proceso de liderazgo de la institución de educación superior privada como estudio de caso; 3 ) el municipio de Tekax cuenta con pobreza, vulnerabilidad, población rural e indígena, lo cual la hace vinculable con el estudio de tesis. Para entender la pobreza que se vive en el estado de Yucatán, particularmente en el municipio de Tekax, es necesario dar una revisión a algunos hechos históricos que fueron los que consolidaron el orden mundial que rige la economía global $y$, por ende, afecta al desarrollo económico social de México.

La globalización es un fenómeno que tuvo sus orígenes con la fundación de América, el capitalismo colonial/moderno, lo que dio origen a la clasificación social de la población mundial sobre la idea de raza. Este concepto de clasificación socio-racial fue la expresión del dominio colonial, el cual permea hasta nuestros días, al poder constatar que la población en pobreza extrema suele ser la población indígena del continente, sin que México, ni el estado de Yucatán sean la excepción (Quijano, 2012).

La dinámica social que surgió a raíz del concepto de raza, produjo en América Latina identidades sociales promotoras de desigualdad social con base en el origen étnico, las cuales se manifestaron como indios, españoles, portugueses y europeos en general. Este factor derivó en una connotación racial en la que se expresaba la dominación de los conquistadores (blancos) sobre los conquistados (indígenas) (Quijano, 2012). Esta situación de dominancia racial, aunque no bajo el mismo contexto, se puede ver refle- jada en las relaciones sociales del Yucatán moderno, en donde el desprecio hacia la población de origen Maya, tiene un impacto en las condiciones de vida desfavorables que estos poseen. El municipio de Tekax es claro ejemplo de ello, cuenta con un índice de pobreza del $68,8 \%$, con una población indígena por encima del $90 \%$, de la cual más del $60 \%$ es maya hablante (Secretaria de Desarrollo Social, 2018).

Aunado al racismo promotor de desigualdad social, otro de los factores que han conducido a la población de América Latina, México y, por ende, del estado de Yucatán a los niveles de pobreza que hoy se conocen, es el modelo eurocéntrico de control y de explotación del trabajo, de la producción, distribución de mercancías, articulados desde la óptica del capitalismo y del mercado mundial. Este modelo económico está diseñado para la explotación de los recursos naturales de los países y regiones menos desarrolladas, sin generar tecnología propia, ni valor agregado para posteriormente ser procesados en las economías desarrolladas, para regresarlos como bienes terminados con valor agregado, a un costo mucho mayor que las materias primas exportadas en un inicio (Quijano. 2012).

Para comprender el concepto de pobreza, cómo se clasifica, cómo se mide y las principales causales que hacen que las comunidades caigan y permanezcan en situación de pobreza y vulnerabilidad, es importante aterrizar una definición del término pobreza.

Según Haughton y Kandler (2009), "la pobreza es la privación del bienestar de manera pronunciada, es decir, la falta de acceso a capacidades básicas para funcionar en la sociedad y de un ingreso adecuado para enfrentar necesidades de educación, salud, seguridad, emprendimiento y derechos básicos". Acorde a la UNESCO (2015), un individuo está en condición de pobreza si su ingreso está por debajo de la línea de pobreza; 
no posee los servicios básicos necesarios, y no cuenta con las capacidades básicas para funcionar en sociedad. Según Galindo y Ríos (2015), la pobreza puede clasificarse en pobreza absoluta y pobreza relativa. La pobreza absoluta es la carencia de dinero para obtener satisfactores básicos, sin considerar aspectos de calidad; mientras que la pobreza relativa se refiere a un estándar de vida, dado en una sociedad que sirva como referencia entre individuos. Kovacevic y Calderón (2014) sostienen que un individuo es pobre, si tiene por lo menos una carencia social, entendiendo como carencia social la falta de acceso a servicios de salud, de seguridad, de educación, alimentarios, sanidad o vivienda digna.

En México, el órgano encargado de medir la pobreza es el Consejo Nacional de Evaluación de la Política de Desarrollo Social (CONEVAL). El CONEVAL mide la pobreza con base en dos dimensiones: bienestar económico y derechos sociales. El bienestar económico se mide con base en una línea de bienestar que indica el ingreso per cápita mínimo necesario para que un individuo adquiera bienes y servicios básicos para la satisfacción de sus necesidades - \$1657,00 pesos corrientes para zonas rurales y $\$ 2591,00$ pesos para zonas urbanas hasta el año 2015- (CONEVAL, 2016a). Mientras que los derechos sociales son salud, educación, seguridad; vivienda y sus servicios (CONEVAL, 2016b).

Con base en lo anterior, el CONEVAL define a la pobreza de dos formas: 1) pobreza multidimensional se refiere a aquellos individuos que tienen un ingreso inferior a la línea de bienestar, y padece al menos de una carencia social; 2) pobreza extrema se refiere a aquellos individuos cuyo ingreso es tan bajo que no puede adquirir los nutrientes necesarios para una vida sana, es decir, se encuentra por debajo de la línea de bienestar mínima, o tiene más de tres carencias sociales (CONEVAL, 2016b).
Esta situación de pobreza y poco desarrollo económico en el municipio de Tekax, se ve reforzada por los bajos niveles de educación que la población tiene (7,6 años de educación promedio para personas mayores a 15 años). Únicamente el 18.51\% de los jóvenes que egresaron del sistema educativo medio superior logra matricularse en las instituciones de educación superior, las cuales se encuentran en la cabecera municipal cuyo nombre es Tekax de Álvaro Obregón. En el municipio solo existen 2 instituciones de educación superior -1 pública y 1 privada-, entre ambas instituciones dan cabida a una matrícula total de 707 alumnos, la tasa de eficiencia terminal promedio de ambas instituciones es del 10.6\%, también es importante considerar, para el contexto, que las principales actividades económicas del municipio de Tekax son la agricultura, la ganadería y el comercio (INEGI, 2017).

Por tal motivo, se aborda a la pobreza y vulnerabilidad de jóvenes yucatecos oriundos del municipio de Tekax, que no pueden acceder a la educación superior y, por ende, sus oportunidades de desarrollo y bienestar, tanto individual como comunitario, se ven afectados negativamente. Chaves (1997) afirma que la educación es uno de los principales móviles sociales para mejorar la condición de vida de la población que pueda acceder a ella.

Derivado de lo planteado en la introducción, se establecen los siguientes cuestionamientos: ¿Cuál es la relación existente entre el desarrollo social y económico con el desarrollo de capital humano e intelectual?, ¿cuál es la relación que existe entre las condiciones de pobreza y vulnerabilidad con la falta acceso a la educación superior en el municipio de Tekax, Yucatán?, ¿cuál es la perspectiva de desarrollo social y económico que tiene el municipio de Tekax, si se apuesta por el desarrollo de capital humano e intelectual por medio de la educación superior? 


\section{Marco teórico}

Este artículo se centra en el estudio sobre el impacto que tiene la falta de acceso a la educación superior de jóvenes en situación de pobreza y vulnerabilidad, en el municipio de Tekax-Yucatán, con respecto al desarrollo económico y social de la comunidad sujeto de estudio. Lo que se busca es demostrar que la educación es uno de los factores que contribuyen a la mejora de las condiciones de vida de los individuos dentro de una comunidad, y que, entre mayor sea el grado educativo que se reciba, mayores serán los beneficios en el mediano y largo plazo para el individuo y para la comunidad.

Cypher y Dietz (2009) sostienen que gracias a la educación toda comunidad, estado o nación se vuelve capaz de apropiarse de una porción de las ganancias provenientes del conocimiento y de los avances tecnológicos, gracias a la acumulación del capital humano generador de crecimiento y desarrollo económico social. El desarrollo económico y social implica el incremento en los índices de productividad en las localidades, en los diferentes sectores de la economía. Si los niveles de vida de la población se ven incrementados, este logro, en gran medida, se da gracias a la mejora de las capacidades de la población de la comunidad por medio del entrenamiento y la educación de la población. Es por esta razón que se vuelve trascendente la acumulación del capital humano para el combate eficaz de la pobreza, no solamente por medio de la acumulación de conocimientos escolarizados, sino, también por medio del aprendizaje a través de la experiencia laboral.

La acumulación del capital humano, combinado con la acumulación de capital físico, así como el uso efectivo de la tecnología crean una sinergia que incrementa la productividad de la fuerza de trabajo y con ello la posibilidad de mejorar la condición de vida de la comunidad. Para los países que cuentan con una alta tasa de población indígena (como es el caso de México y particularmente la zona de estudio de esta investigación), se hace necesario no únicamente lograr esta la sinergia, sino también considerar, desarrollar técnicas y tecnologías autónomas basadas en las capacidades y creencias de la población indígena. Una de las principales barreras que entorpecen el desarrollo económico y social de una comunidad son los sistemas educativos obsoletos y subdesarrollados, que no logren dar cabida a los alumnos que se van titulando de grados inferiores a los grados siguientes.

Según Cypher y Dietz (2009), toda sociedad que tenga un interés legítimo para mejorar las condiciones de vida de su población, debe perseguir los siguientes objetivos: igualdad de oportunidades; incrementos en los niveles salariales, que permitan incrementar los niveles de vida de la población; equidad en la distribución del ingreso y la riqueza; democracia y una alta participación ciudadana en las decisiones de la comunidad; una participación más decidida del rol de la mujer en la economía y la política del lugar; acceso a servicios de salud; redes de protección para población en condición de vulnerabilidad que prevengan su potencial caída a condición de pobreza; la promoción de ambientes saludables tanto física como psicológicamente; instituciones públicas transparentes, eficientes, competentes y bien administradas; alta competitividad empresarial.

Acorde al Programa de Desarrollo de las Naciones Unidas, que establece el postulado: el desarrollo humano va más allá del incremento del ingreso nacional bruto, se trata de crear ambientes propicios, en los cuales las personas puedan desarrollar su potencial para generar vidas productivas $y$ creativas acorde a sus necesidades e intereses, esto solo será posible por medio al 
acceso a la educación en todos sus niveles. "La gente es la verdadera riqueza de las naciones" (Naciones Unidas, 2001).

La educación superior es importante porque constituye un mecanismo de movilidad social; sin embargo, si las instituciones de este nivel educativo mantienen los patrones de acceso que favorecen estudiantes de un solo nivel socioeconómico y de una misma cultura, la consecuencia es la inequidad, especialmente para poblaciones en situación de vulnerabilidad económica, étnica o cultural. Para que pueda lograrse un acceso equitativo que permita a todos los estudiantes ingresar y tener éxito en la educación superior, es necesario que los sistemas educativos actuales se redirijan a la brevedad posible e implementen cambios significativos para favorecer el acceso de todos los estudiantes, especialmente aquellos que son vulnerables (Haveman y Smeeding, 2006).

Ayres (1995) sostiene que la educación es la difusión del conocimiento y las habilidades, por ende, expandir las oportunidades de integración a la educación superior a más personas dentro una comunidad es apostarle a la acumulación del capital humano en el mediano plazo y a la acumulación del capital intelectual en el largo plazo, lo que a su vez redundará en el fomento del progreso económico y social. Ayres menciona que el factor más importante en la vida económica de los individuos es lograr el máximo grado de estudios posible dentro de su comunidad; del mismo modo, manifiesta que la tecnología y la educación son las bases para la creación de estructuras institucionales apropiadas que den soporte al progreso humano.

El capital intelectual se logra cuando la academia y el sector empresarial generan estrategias formativas de manera conjunta o individualizada para educar, capacitar y entrenar personas generando espacios de aprendizaje constantes y continuos en búsqueda de la innovación para la resolución de problemas.
La aplicación de la ley del 80-20, en e uso del tiempo organizacional, es decir, 80\% del tiempo ocuparlo en la ejecución de las tareas cotidianas que ayuden a mantener los niveles de efectividad organizacional; y el 20\% restante ocuparlo para la creación de proyectos de innovación procesal, tecnológica, de diseño y desarrollo de soluciones que generen valor agregado y con el paso del tiempo ventajas competitivas, este modelo de aprendizaje organizacional es conocido como el proceso de desaprender para aprender, solo es viable bajo el modelo institucional de organizaciones que aprenden u organizaciones inteligentes propuesto por Peter Senge (2014).

El modelo de aprendizaje organizacional funciona mediante la implementación de 5 disciplinas: aprendizaje en equipo, sosteniendo la tesis que el conocimiento es socializable y debe ser compartido para generar progreso; pensamiento sistémico, que permita entender la globalidad y el contexto en el cual se está inmerso para generar soluciones ad hoc; dominio personal, que permita la autogestión, la autorregulación, la automotivación y una fuerte tolerancia a la frustración; modelos mentales para el planteamiento de soluciones racionales que permitan el mapeo de la solución, desde que la necesidad es identificada, el diseño de la solución es creado, el desarrollo de la solución es ejecutado, la implementación llevada a cabo, el monitoreo de la misma para medir su efectividad es retroalimentado y el nivel de satisfacción de quienes están utilizando la solución es verificado; y, una visión compartida, que permita tener un punto de llegada común entre todos los colaboradores que les oriente para trabajar sinérgicamente en un fin o varios fines en común. Estas disciplinas ayudan a comprender la complejidad, adquirir compromisos, asumir responsabilidades, buscar un constante autocrecimiento, crear sinergias a través del 
trabajo colaborativo. Este modelo de aprendizaje organizacional puede efectuarse en todo tipo de organizaciones, sin embargo, las organizaciones que mejor han aplicado este modelo son las instituciones educativas, de investigación y desarrollo; así como las organizaciones de alta tecnología.

Acorde a estudios realizados a economías del este asiático, el Banco Mundial (1993) determinó que la formación de capital humano y capital intelectual en estas sociedades han sido factores determinantes para el desarrollo económico. Por esto es importante que la intervención de las políticas gubernamentales jueguen un papel fundamental para fomentar la inversión tanto pública como privada en el sector educativo. Por otro lado, se han podido observar algunos beneficios secundarios con el incremento de la participación de la población en los niveles educativos más altos. Estos beneficios se ven reflejados, no solo en una sustancial mejora en la ejecución de tareas, que conlleva en mejoras en cuanto al alcance de objetivos se refiere, sino, que las personas con mayor educación son capaces de crear sinergias y con ello incrementar la efectividad, productividad y competitividad de manera colaborativa con sus compañeros de trabajo - la famosa fórmula de la sinergia $1+1=3-$.

De tal suerte que la inversión realizada por los gobiernos en educación, es inversión en capital humano, intelectual y social, que es tan o más importante que la inversión en infraestructura física para el crecimiento económico y para el desarrollo humano. En muchos de los casos, y sobre todo en economías menos desarrolladas - principalmente latinoamericanas-, esta inversión en infraestructura social ha sido demeritada, lo que ha sido causal significativa para la existencia de brechas de desarrollo económico y humano entre las naciones y aún dentro de las mismas naciones de manera regional (Rodrik, 1994). Tal es el caso de México, y particularmente del estado de Yucatán, en donde se puede observar que las zonas con mayor infraestructura social y física, tienen mayores oportunidades de crecimiento y mejores condiciones vida (CONEVAL, 2016a).

Lucas (1988) manifiesta que la productividad de todo individuo que ha invertido en desarrollar su capital humano suele ser más alta que aquellos que no lo han hecho; del mismo modo, cuando un individuo que ha invertido en el desarrollo de su capital humano, se asocia con otros que tienen más desarrollado el capital humano, su capacidad de aprendizaje se ve potenciada, su capacidad de contribución se incrementa. Todo esto, según Senge (2012), conlleva a la creación de círculos virtuosos de aprendizaje organizacional que potencia la innovación en la resolución de problemas por medio del capital intelectual desarrollado. A mayor educación, mayores beneficios personales y sociales, Rowen (1996) y Amsden (2001) mencionan que toda economía interesada en lograr un desarrollo económico sostenible, tendrá que invertir en el desarrollo del capital humano por medio del acceso a la educación: tal es el caso de la India, en donde el correcto diseño de políticas públicas, encaminadas a la inversión en infraestructura educativa universitaria en el ámbito de la ingeniería tecnológica, ha puesto al país en la posibilidad de observar cambios positivos en las condiciones de vida de su población, gracias al desarrollo de tecnología propia exportable a otros países (Hamm, 2007).

Por otro lado, un medio eficaz para disminuir la pobreza en zonas rurales, es la inversión en educación significativa para las personas que viven y dependen del campo. El empoderamiento de los jóvenes rurales ayuda a incrementar la productividad en el campo, y a disminuir las tasas migratorias del campo hacia los centros urbanos. Acompañada de esta infraestructura escolar rural, debe desarrollarse infraestructura en otro tipo de servi- 
cios como es el de acceso a la salud, este tipo de medidas contribuye de manera efectiva al desarrollo socioeconómico de las zonas rurales (Cypher \& Dietz, 2009).

Para que esto sea una realidad se hace necesario un verdadero compromiso en el diseño de políticas públicas en educación, encaminadas a desarrollar el campo por medio del aprendizaje de mejores técnicas de ejecución del ciclo agrario, con ayuda de tecnología de punta. Esto tendría un impacto positivo en las economías de los países menos desarrollados (Banco Mundial, 2003). Una investigación realizada por el Departamento de Economía y Asuntos Sociales de las Naciones Unidas (2000) evidenció que el retorno de la inversión, por cada dólar, en la investigación agrícola en países en desarrollo, es de 80 centavos, lo que representa el doble e incluso el triple de los retornos observados en economías desarrolladas.

Según Adler (1987), en algunas ocasiones los países menos desarrollados han invertido en la formación de ingenieros y científicos en el extranjero; sin embargo, el tipo de educación que han recibido no les ha resultado útil para solucionar los problemas que aquejan a sus países. Esta situación ha contribuido a la fuga de cerebros, que lejos de contribuir al desarrollo del país que los envió para mejorar sus capacidades, se convierte en un gasto para la nación.

Situaciones similares se pueden presentar en los jóvenes de comunidades rurales pequeñas que tienen la oportunidad de estudiar en sus comunidades, en donde su educativa sea de carreras con disciplinas y contenidos que no sean vinculables con las actividades económicas de la comunidad. Por un lado alienta la migración de estos jóvenes egresados de sus comunidades a centros urbanos buscando oportunidades de empleo acorde a los estudios que realizaron; o, por otro lado, bien habría una matriculación poco significativa por parte de los jóvenes a los cuales se está buscando formar. El primer escenario pondría en serias complicaciones a los pequeños productores de cultivos comerciales, porque la mano de obra para trabajar el campo se vuelve escasa y menos cualificada; mientras que en el segundo escenario, la permanencia del centro educativo de la comunidad perdería su viabilidad de subsistencia.

Independientemente del sector de la economía que se trate, una fuerza de trabajo debidamente educada es imprescindible, esto con el fin de lograr el desarrollo humano de una comunidad; las economías que no observen este principio, estarán truncando su camino hacia el desarrollo económico y social. La acumulación del capital humano, intelectual y social son factores clave para el desarrollo. En estudios realizados en diversos países considerados como economías desarrolladas, se ha podido observar que el éxito de estas naciones ha sido apostar a tres variables: mayor y mejor acceso a la educación en todos los niveles, la profesionalización de la población; el incremento de los ingresos de la población; y, con ello, mejorar el nivel de vida de la población (Cypher y Dietz, 2009).

Sen (1999) sostiene que el desarrollo económico se logra por medio de la expansión de las capacidades de la población de una comunidad; sin embargo, menciona que la inversión pública hecha en el sistema educativo, en algunos países, no necesariamente se traduce en un inhibidor de la pobreza, ya que esto depende en gran medida de la efectividad en el manejo de los recursos por parte de la dependencia responsable.

Estudios del Banco Mundial (1993) muestran la contribución que la educación tuvo en el desarrollo económico y social de los países que tomaron decisiones contundentes en cuanto a inversión en educación, para desarrollar su capital humano, intelectual y social. Este grupo de paises compren- 
de el bloque de los Tigres del Pacífico durante el periodo entre los años de 1960 a 1985, con los siguientes resultados: Tailandia $87 \%$, Hong Kong 86\%, Indonesia 79\%, Singapur 75\%, Malasia 73\%, Taiwán 69\%, Corea del Sur $67 \%$ y Japón 58\%. Estos porcertajes fueron realidad gracias a políticas públicas diseñadas en estos países, con la finalidad de invertir en el acceso a la educación en todos los grados escolares: desde la educación básica hasta la educación superior apoyada por planes de capacitación y entrenamiento de las empresas con la finalidad de desarrollar las competencias de sus colaboradores. Lo que según Cypher y Dietz (2009), demuestra como la inversión en educación y profesionalización, se traduce en una mayor acumulación de capital humano e intelectual, misma que se refleja en capital social y bienestar para las sociedades.

Estudios realizados en Asia del Este han demostrado que la inversión hecha por los gobiernos de países como Corea del Sur, Malasia y Tailandia, para dotar de acceso a su población a educación de calidad, contribuyó a disminuir la desigualdad de ingresos en sus economías y con ello incrementar el crecimiento y desarrollo económico en sus países. Otro fenómeno observado en estas economías, es que, de manera particular, la inversión hecha para brindar acceso a la educación superior a su población, desarrollo trabajadores más capaces, facultados para obtener mejores empleos, con mayores ingresos, lo que causó una baja sensible en la inequidad de los ingresos (Birdsall y Sabot, 1994).

En la actualidad, una de las principales ventajas competitivas que las economías asiáticas están explotando, es el desarrollo del capital humano e intelectual para promover el consumo interno, bajo la tesis de entre mejor cualificada esté la población del país, se reflejará en mayor productividad, que a su vez se verá reflejado en mayores ingresos, que implica mayor poder de compra, con el consecuente incremento de demanda agregada de productos y por ende un mercado interno sólido capaz de dar cabida a más empresas, que generan más productos y con ello mayor empleo, como se ha podido observar en las últimas décadas en países como Taiwán y Corea del Sur (Cypher \& Dietz, 2009).

Según el Banco Mundial (2003), se ha generado una campaña mundial, que ha establecido la necesidad de lograr el acceso universal a la educación básica, así como promover el acceso a la educación superior como motor del crecimiento y desarrollo económico. En algunos países que han tenido resultados sobresalientes en esta materia son China, India, Singapur, Sri Lanka, Vietnam, Costa Rica y Panamá. Estas naciones han conseguido sustanciales crecimientos económicos en las últimas décadas, del mismo modo eso ha contribuido a mejorar los niveles de vida de la población en estos países.

Como parte de los objetivos del milenio, planteados por las Naciones Unidas, se establecieron como primordiales la reducción de la pobreza por medio de la ampliación del acceso a los servicios de salud; así como la expansión de oportunidades de acceso a la educación para la población en condición de pobreza y vulnerabilidad. Esta visión unificada reconoce que todas las economías del planeta están interconectadas, por tanto, el problema de pobreza concierne a todas las naciones. Para el combate eficaz de la pobreza, Cypher y Dietz (2009) mencionan que dicho combate tiene que estar acompañado por una serie de valores, que tienen que ser observados por la sociedad como la oportunidad de conseguir empleos significativos por parte de la población; contar con alimentos suficientes, así como, con los recursos necesarios para gozar de seguridad y tranquilidad; tener la oportunidad de acceder a educación de calidad, que permita desarrollar capacidades útiles para la vida 
personal y profesional; niveles de seguridad social aceptables; democracia y participación ciudadana en los temas nacionales; trato justo y equitativo al sistema de justicia, e inclusión y respeto a la condición humana.

\section{Metodología}

El método empleado para esta investigación fue una revisión y análisis de fuentes de información primaria como artículos científicos que hablan sobre temas de pobreza, vulnerabilidad, desarrollo económico y social; secundaria, en la que constan libros concernientes a temas de economía, y terciaria que recoge reportes de organismos gubernamentales y no gubernamentales tanto nacionales como internacionales, que aportan datos estadísticos sobre pobreza, vulnerabilidad, acceso a la educación y es- trategias para combatir a la pobreza y la inequidad social.

\section{Análisis y resultados}

La pobreza es una consecuencia multifactorial cuyas causas son, entre otras, la disparidad de los ingresos, la mala distribución de la riqueza, así como la falta de acceso a la educación, que permita a la población desarrollar las capacidades necesarias para desarrollarse adecuadamente y con ello salir del círculo de la pobreza y vulnerabilidad (Cypher y Dietz, 2009).

México tiene varios pendientes por mejorar, el país se encuentra en el puesto número 58 del ranking mundial de progreso social con un puntaje promedio de 70,42 puntos. Este indicador se conforma de 3 subíndices (los cuales se derivan en otros subíndices).

Tabla 1. Índice de Progreso Social

\begin{tabular}{|c|c|c|}
\hline $\begin{array}{l}\text { Necesidades humanas básicas } \\
(80,85 \text { puntos })\end{array}$ & $\begin{array}{l}\text { Fundamentos de bienestar } \\
\text { social ( } 73,85 \text { puntos) }\end{array}$ & $\begin{array}{l}\text { Oportunidades } \\
\text { (56,56 puntos) }\end{array}$ \\
\hline $\begin{array}{l}\text { Nutrición y servicios de salud básicos } \\
(93,12) \\
\text { Agua y servicios sanitarios }(94,22) \\
\text { Acceso a Servicios de electricidad }(87,81) \\
\text { Seguridad }(48,27)\end{array}$ & $\begin{array}{l}\text { Acceso a educación básica } \\
(84,79) \\
\text { Acceso a la información y comu- } \\
\text { nicación }(62,84) \\
\text { Salud y bienestar }(62,16) \\
\text { Calidad ambiental }(85,62)\end{array}$ & $\begin{array}{l}\text { Derechos humanos }(73,98) \\
\text { Libertad de elegir }(62,02) \\
\text { Inclusión social }(44,41) \\
\text { Acceso a educación superior } \\
(45,80)\end{array}$ \\
\hline
\end{tabular}

Tabla de elaboración propia con datos de Social Progress Index, (2018b).

Para interpretar los índices reflejados en la tabla 1 , se debe analizar de manera minuciosa y crítica debido a que la calificación máxima que se puede obtener en cada subíndice, así como en el índice global es de 100 puntos. Sin embargo, el juicio de valor de la calificación (encima del promedio, promedio o por debajo del promedio) se hace con base en el puesto dentro del ranking que ocupa el país con respecto del resto de los países de un total de 146 evaluados. Por ende, aunque México en el rubro de Acceso a la educación superior aparece como un país sobresaliente por ocupar la posición número 38 de 146 (Social Progress Index, 2018b); su desempeño real es muy pobre y la misma calificadora así lo manifiesta (como una de las debilidades del país), con un puntaje de 45,80 de 100 puntos posibles (Social Progress Index, 2018a).

Si bien se ha mencionado que una de las variables que conducen a la pobreza es la falta de acceso a la educación como derecho social (CONEVAL, 2016b), ciertamente no es 
la única variable, se trata de un problema multifactorial, que va intimamente ligado al crecimiento económico. Teóricamente, altas tasas de crecimiento económico tienden a reducir la pobreza absoluta (Ravallion, 2013), sin embargo, este efecto es menor cuando la distribución del ingreso es desigual (Bourguignon, 2004); es decir, en países con alta desigualdad, los pobres suelen beneficiarse menos del crecimiento económico, por consiguiente, la pobreza se ve menos afectada por las altas tasas de crecimiento (Ravallion, 2013).

En este tenor, México es uno de los países más desiguales del mundo, es la economía número 14 en cuanto a tamaño y volumen económico, pero tiene 45 millones de personas viviendo en situación de pobreza, lo que sumerge al país en un círculo vicioso de desigualdad, bajo crecimiento económico y pobreza. México se encuentra dentro del $25 \%$ de los países con mayores niveles de desigualdad de la OCDE, los principales causales de la desigualdad en México son el 1\% de la población recibe el $21 \%$ de ingresos de todo el país; los ricos se hacen más ricos, en la actualidad la fortuna de los cuatro hombres más ricos de México equivale al 9.5\% del PIB -en el 2002 era del 2\% del PIB-; existen sectores privilegiados por falta de competencia económica, una deficiente regulación, y un régimen fiscal que favorece a los más ricos (Forbes, 2015).

En México, la situación educativa es compleja debido al limitado acceso de los egresados de bachillerato a educación superior. De acuerdo con datos de (Fuentes, 2017), solamente 3.6 millones (31\% de estudiantes) que concluyen la educación media superior ingresan a las universidades e institutos de educación superior en el país. En el caso de Yucatán, el porcentaje de ingreso a este nivel, según datos de la Secretaría de Educación Pública (Fuentes, 2017), es solo del $27,2 \%$, esto indica que el $72,8 \%$ de los estudiantes egresados de bachillerato no lo- gra tener acceso a educación superior en el Estado de Yucatán.

Las estadísticas son más alarmantes cuando se analizan los datos tomando en cuenta el nivel socioeconómico y cultural de los estudiantes. Como indican datos presentados por Pérez (2010), el porcentaje de estudiantes en condición de marginalidad que ingresan a la educación superior en México es del 1,8\%; además, Pérez afirma que de este $1,8 \%$ de alumnos en condiciones de pobreza y vulnerabilidad el $7.7 \%$ son precisamente estudiantes que provienen de comunidades indígenas de zonas rurales.

Para el caso de Yucatán, de la población de alumnos en condición de pobreza y vulnerabilidad, el $40,6 \%$ son provenientes de comunidades indígenas de zonas rurales, esta falta de acceso a la educación superior en comunidades en situación de pobreza como lo es el municipio de Tekax-, se debe a la falta infraestructura suficiente, la falta de recursos económicos por parte de los jóvenes recién egresados del sistema de educación medio superior (INEGI, 2015), así como, falta de visión y liderazgo generador de oportunidades de desarrollo de capital humano por parte de las instituciones de educación superior que cuentan con algún campus al interior del Estado. Como consecuencia, se refleja la incapacidad de las empresas existentes en el municipio para generar capital intelectual debido a su naturaleza y características, lo que dificulta el desarrollo de capital social.

En un intento para incrementar el acceso de la población a la educación superior, en los últimos 30 años se han creado más instituciones de educación superior en todos los estados. Para el caso de Yucatán, como se aprecia en la tabla 2, el incremento de universidades e institutos de educación superior ha sido del 330\%; del mismo modo, también se han creado centros de investigación que buscan contribuir al desarrollo 
económico y social por medio de estudios e investigaciones dentro del Estado.

Tabla 2. Número de instituciones de educación superior en un periodo de 30 años

\begin{tabular}{|c|c|c|c|}
\hline Instituciones de educación superior & Hasta 1988 & $1988-1998$ & $1998-2018$ \\
\hline Instituciones de Educación Superior Públicas & 10 & 10 & 6 \\
\hline Instituciones de Educación Superior Privadas & 20 & 35 & 18 \\
\hline Centros de Investigación & 3 & 4 & 13 \\
\hline
\end{tabular}

Tabla de elaboración propia con datos de (Subsecretaria de Educación Pública, 2018); González, González y Aguilar, 2010).

Aunque hubo un incremento en la creación de instituciones de educación superior públicas, ese aumento ha sido incipiente, uno de los mayores se observa en el caso de las universidades privadas; asimismo, se aprecia una tendencia por parte de estas instituciones privadas para abrir nuevos programas en el interior del Estado, en comunidades con diferente nivel socioeconómico y en las cuáles existen una mayor proporción de estudiantes maya hablantes.

Por otro lado, es innegable la contribución que la educación superior ha traído al estado de Yucatán, formando profesionales en diferentes disciplinas que en su mayoría han sido en el ámbito de las ciencias sociales, seguidas de las áreas económico-administrativas, en el tercer sitio las ciencias médicas y al final se encuentran las áreas de las matemáticas y las ingenierías. Sin embargo, el impacto de este esfuerzo para dar acceso a la educación superior, se ha centralizado en la zona noroeste del Estado, en donde se encuentra la ciudad de Mérida que es la capital; este efecto también se ha visto reflejado en un desequilibrio en el desarrollo económico y social del eEstado el cual también se ha focalizado en la zona noroeste del estado (González, González y Aguilar, 2010).

En el caso de Yucatán, al igual que en el resto del país, la desigualdad, la pobreza y la vulnerabilidad son de los grandes pendientes que no han podido resolverse de manera satisfactoria; aunque según la Subsecretaría de Planeación Evaluación y Desarrollo Regional, del 2010 al 2016 se han tenido avances en el combate a la pobreza, logrando una reducción del 5,91\% de la pobreza en la población, como lo muestra la tabla 3 "Indicadores de pobreza y desigualdad, 2010-2016".

Tabla 3. Indicadores de pobreza y desigualdad 2010-2016

\begin{tabular}{|l|l|l|l|l|}
\hline \multicolumn{1}{|c|}{ Indicador } & \multicolumn{1}{c|}{$\mathbf{1}$ 2010 } & \multicolumn{1}{c|}{$\mathbf{2 0 1 2}$} & \multicolumn{1}{c|}{$\mathbf{2 0 1 4}$} & \multicolumn{1}{c|}{$\mathbf{2 0 1 6}$} \\
\hline Población en pobreza & 958,546 & 996,874 & 957,908 & 901,864 \\
\hline Coeficiente de Gini** & 0,462 & 0,461 & 0,511 & 0,452 \\
\hline
\end{tabular}

Fuente: Informe anual sobre la situación de pobreza y rezago social 2018 (Secretaría de Desarrollo Social, 2018).

Según datos proporcionados por González, González y Aguilar (2010), la región sur del Estado está conformada por 17 municipios, (entre los cuales se encuentra el municipio de Tekax). La siguiente es la situación en cuanto a desarrollo de actividades y sectores económicos se refiere: existen 654 empresas de las cuales el 48\% llevan a cabo actividades propias del sector primario; $41 \%$ son empresas con actividades del sector terciario, y solo el $11 \%$ son empresas con actividades dentro del sector secunda- 
rio. La región sur es la menos poblada, pero también es la que menor ingreso per cápita genera en el territorio. Curiosamente, la región sur es la segunda con mayor porcentaje de población urbana con un $41,5 \%$ de la población, en teoría, este último punto debería permitir facilidades en la transferencia de tecnología a la población y a las empresas del municipio.

Con respecto al municipio de Tekax, la principal actividad económica son la agricultura, ganadería y comercio. Durante el ciclo escolar 2015-2016, en Tekax, el comportamiento estadístico del alumnado que demandó estudios de técnico superior universitario o profesional asociado en la modalidad escolarizada fue el siguiente: alumnos inscritos 693, alumnos egresados 269, alumnos titulados 163, en las disciplinas de ciencias naturales, exactas y de la computación; ciencias sociales, administración y derecho; ingeniería, manufactura y construcción, y servicios. Con respecto a los alumnos que demandaron estudios de licenciatura, el comportamiento estadístico durante el mismo ciclo escolar fue de 0 alumnos, misma situación para los alumnos que demandaron estudios de posgrado en el mismo ciclo escolar (INEGI, 2017).

Con respecto al indicador concerniente al rezago educativo, el municipio mejoró $6,6 \%$, sin embargo, el índice de rezago sigue siendo alto, del $26,5 \%$, con una tasa de acceso a la educación superior del 18,51\% con respecto de alumnos egresados del sistema medio superior y una tasa de titulación de estudios de licenciatura del 10,6\% hasta 2015 (Secretaria de Desarrollo Social, 2018). En conclusión, esto está de manifiesto con la existencia de tan solo dos instituciones de educación superior en la cabecera municipal, la Universidad Tecnológica Regional del Sur y la Universidad sujeto de estudio. (Secretaría de Educación Pública, 2015).

\section{Conclusiones}

¿Cuál es la relación existente entre el desarrollo social y económico con el desarrollo de capital humano e intelectual?

Como se refirió a lo largo del artículo, tomamos como base la experiencia y las evidencias encontradas sobre los casos de éxito en los países del este asiático para reflejar estarelación. En los que estos gobiernos apostaron al diseño de políticas públicas que garantizaron la universalización de la educación básica, media básica y media superior para su población; al mismo tiempo, este hito logrí impulsar a la matriculación y eficiencia terminal en el sistema educativo superior para formar capital humano en diversas disciplinas, pero más enfocadas a los estudios de ciencias e ingenierías. Gracias a este refuerzo en estas materias, dio como resultado una fuerza laboral tecnificada, capaz, comprometida, disciplinada, eficiente y productiva, dispuesta a mejorar su situación de vida y con ello contribuir al crecimiento y desarrollo económico de sus respectivos países.

Sin embargo, no se puede dejar de lado los esfuerzos de las organizaciones privadas con fines de lucro, quienes también apostaron por la competitividad empresarial por medio del desarrollo de capital intelectual. Esta ha creado polos de investigación y desarrollo de productos tecnológicos con alto valor agregado por medio del aprendizaje organizacional, fomentando la creatividad colaborativa, el aprendizaje innovador en todas sus líneas.

De esta forma, tanto el gobierno como la iniciativa privada contribuyeron de manera conjunta y coordinada con planes de desarrollo nacional y políticas públicas encaminadas a la formación de capital humano e intelectual. Así se logró una mayor equidad salarial y social en periodos de tiempo de mediano a largo plazo en estas economías. 
¿Cuál es la relación existente entre las condiciones de pobreza y vulnerabilidad con la falta acceso a la educación superior en el municipio de Tekax, Yucatán?

De igual manera, según la evidencia encontrada a lo largo del presente artículo, las condiciones de pobreza y vulnerabilidad en el municipio de Tekax, Yucatán no son casualidad, sino causalidad. Una de las causalidades tiene que ver con el rezago educativo que se vive en el municipio: del $100 \%$ de los alumnos que logran egresar del sistema educativo medio superior en Tekax, solo el $18 \%$ se logra matricular en las instituciones de educación superior de la localidad.

Este rezago educativo se ve reflejado en el tipo de actividades económicas y empresariales que se han logrado establecer en el lugar, cuyas características principales son empresas principalmente enfocadas a sectores primarios (agricultura y ganadería) y terciarios (comercio al por menor y servicios profesionales); de tamaño micro, en su mayoría bajo la modalidad de personas físicas, con poco o nulo desarrollo tecnológico; los productos y servicios que se ofrecen son de bajo a nulo valor agregado; las empresas de Tekax suelen ser de autoempleo, encaminadas para lograr la supervivencia de los miembros de la familia, y difícilmente pueden generar más de 10 empleos para convertirse en pequeñas empresas.

¿Cuál es la perspectiva de desarrollo social y económico que tiene el municipio de Tekax, si se apuesta por el desarrollo de capital humano e intelectual por medio de la educación superior?

A modo de inferencia y con base en lo que se encontró en esta investigación, tomando como referencia lo sucedido con el milagro asiático, podría haber un desarrollo económico y social en el mediano y largo plazo en el municipio de Tekax. Esto sería posible si se crearan las condiciones para que la población tenga acceso educativo en todos los niveles escolares en la comunidad, para que cada vez más jóvenes cuenten con mejores capacidades que les permitan desarrollarse como emprendedores y trabajadores.

Sería importante que la oferta educativa se encaminara a los sectores y actividades económicas de la región, para de esa manera inhibir la migración de los egresados a las zonas urbanas más grandes. Este programa educativo tendría que reforzarse con inversión en infraestructura social, física y tecnológica, que hagan más atractiva la forma de vida dentro del municipio de Tekax. Para esto se deben identificar actividades económicas con potencial de desarrollo, e invertir en ellas para generar mayores y mejores oportunidades de empleo y estudio, este punto también sería inhibidor de la migración de la población juvenil a otras ciudades; promover mecanismos que permitan la subsistencia de la población. Por último, y no menos importante, debería resaltarse la dignificación de la población y el derecho de libertad de elección en cuanto a oportunidades de crecimiento y desarrollo, tanto personal como profesional con impactos positivos en la comunidad.

\section{Referencias}

Adler, E. (1987). The power of ideology: The quest for technological autonomy in Argentina and Brazil. Berkeley, California, USA: University of California Press.

Amsden, A. (2001). The rise of "the Rest": Challenges to the West from late-industrializing economies. Oxford, United Kingdom: Oxford University Press. Retrieved from https://www.worldcat.org/title/ rise-of-the-rest-challenges-to-the-westfrom-late-industrializing-economics/ oclc/488381208/viewport

Ayres, C. (1995). Economic Development: An institutionalist perspective. In J. Dietz (ed.), Latin America's Economic Development, 2nd edn, (pp. 89-97), London, England: Lyne Rienner Publishers. 
Birdsall, N., Ross, D., \& Sabot, R. (1994). Inequality as a Constraint on Growth in Latin America, Development Policy, 1(5). Retrieved from https://search.proquest.com/openvi ew/37fa94e66a9e0e146948db014d7135 d7/1?pq-origsite=gscholar $\& c b l=2035113$

Chaves, A. L. (1997). Economía y educación. Revista Educación, 21(1). Recuperado de https://revistas.ucr.ac.cr/index.php/educacion/article/view/7292

Cypher, J. M., \& Dietz, J. L. (2009). The Process odf Economic Development. 3rd ed. New York, New York, USA: Routledge Taylos \& Francis Group.

Consejo Nacional de Evaluación de la Política de Desarrollo Social. (2016a). Entidades Federativas. Recuperado de https://www. coneval.org.mx/coordinacion/entidades/ Yucatan/Paginas/principal.aspx

Consejo Nacional de Evaluación de la Política de Desarrollo Social. (2016b). Medición de la Pobreza. Recuperado de https://www. coneval.org.mx/Medicion/Paginas/Pobrezalnicio.aspx

Department of Economic and Social Affairs. (2000). World Economic and Social Survey 2000. New York, New York, USA: United Nations. Retrieved from https:// www.un.org/development/desa/dpad/ publication/world-economic-and-socialsurvey-2000/

Forbes Staff (Junio 24, 2015). 7 datos que muestran la desigualdad extrema en México. Forbes. Recuperado de https://www.forbes.com.mx/7-datos-que-muestran-ladesigualdad-extrema-en-mexico/

Fuentes, M. (08 de agosto de 2017). México Social. Excelsior. Recuperado de https://www.excelsior.com.mx/nacional/2017/08/08/1180263

Galindo, M. y Ríos, V. (2015). Pobreza en serie de estudios económicos, 1. México D.F.: México ¿cómo vamos?. Recuperado de https://scholar.harvard.edu/files/vrios/ files/201508_mexicopoverty.pdf?m=

Hamm, S. (March 19, 2007). The trouble with India. Bloomberg Businessweek. Retrieved from https://www.bloomberg.com/news/ articles/2007-03-18/the-trouble-withindia

Haughton, F., \& Khandker, S. (2009). Handbook Povertry + Inequaty. Washington, USA: The World Bank.

Haveman, R., \& Smeeding, T. (2006). The Role of Higher Education in Social Mobility. The Future of Children, 16(2), 125-150. Retrieved from https://www.jstor.org/stable/3844794

Instituto Nacional de Estadística y Geografía. (2017). Anuario estadístico y geográfico de Yucatán 2017. Recuperado de http:// internet.contenidos.inegi.org.mx/contenidos/Productos/prod_serv/contenidos/espanol/bvinegi/productos/nueva_estruc/ anuarios_2017/702825095116.pdf

Kovacevic, M., \& Calderón, C. (2014). UNPD`s Multidimensional Poverty Index. New York, New York, USA: UNDP.

Lucas, R. E. (1988). On the mechanics of economic development. Journal of Monetary Economics, 22(1), pp 3-12. Retrieved from https://www.sciencedirect.com/science/ article/abs/pii/0304393288901687

Pérez, M. (2010). México: Exclusión educativa en un contexto de pobreza y desigualdad. Trabajo presentado en el Seminario de Educación Superior de la UNAM. Distrito Federal, México: Presentación recuperado de https://www.ses.unam.mx/curso2012/pdf/PerezGarcia_M5S1.pdf

Quijano, A. (2012). Colonialidad del poder, eurocentrismo y América Latina. Praxis Conciencia y Libertad, Recuperado de https:// marxismocritico.com/2012/07/19/colonialidad-del-poder-eurocentrismo-y-america-latina/

Ravallion, M. (2013). The idea of antipoverrty policy. Cambridge, United Kingdom: National Bureau of Economic Research.

Rodrik, D. (1994). King Kong Meets Godzilla: The World Bank and The East Asian Miracle. In A. Fishlow, et al., Miracle or design?: Lessons from the East Asian experience, (pp 13-53). Washington, DC, USA: Overseas Development Council. Retrieved from https://econpapers.repec.org/paper/ cprceprdp/944.htm 
Rowen, H. S. (1996). World Wealth Expanding: Why a rich, democratic and (perhaps) peaceful era is ahead. In R. Landau, T. Taylor, \& G. Wrigth (eds.). The Mosaic of economic growth, Stanford, California, USA: Stanford University Press.

Secretaría de Educación Pública. (2015). Panorama de la Educación Superior en Yucatán. Recuperado de http://www.google.com. $\mathrm{mx} / \mathrm{url}$ ? sa $=t \& r c t=j \& q=\& e s r c=s \&$ sour $c e=$ web $\& c d=3 \& c a d=r j a \& u a c t=8 \& v e d$ $=2$ ahUKEwiToKeaodHdAhVEO4MKHZa 5BJkQFjACegQIBXAC\&url=http\%3A\% 2F\%2Fwww.pides.mx\%2Fpanorama esmex_2015_2016\%2F31_yucatan. panorama_esmex_m.pdf\&usg=AOv Vaw21bL7vUP2GmpmlQ_slz9Qa

Secretaría de Desarrollo Social. (2018)._Informe anual sobre la situación de pobreza y rezago social 2018: Yucatán, Tekax. Recuperado de https://www.extranet.sedesol. gob.mx/pnt/Informe/informe_municipal_31079.pdf

Sen, A. (December 20, 1990). More than $100 \mathrm{mi}-$ llion women are missing, New York Review of Books. Recuperado de https://www. nybooks.com/articles/1990/12/20/morethan-100-million-women-are-missing/

Senge, P., Roberts, C,. Ross, R., Smith, B. y Kleiner, A. (2012). La quinta disciplina: el arte y la práctica de la organización abierta al aprendizaje. Buenos Aires, Argentina:Granica
Social Progress Index. (2018a). 2018 Social Progress Index. Recuperado de https://www. socialprogress.org/

Social Progress Index. (2018b). 2018 México. Recuperado de https://www.socialprogress. org $/$ ?tab $=2 \&$ code $=$ MEX

Subsecretaría de Educación Pública. (2018). Instituciones de Educación Superior. Recuperado de https://www.ses.sep.gob.mx/intranet1/aplicacion/index.php?r=catalogos\% 2Finstituciones\%2Flistalnstituciones\&In stituciones\%5Bies_particulares\%5D=ies_ particulares\&Instituciones $\% 5 B$ nom breins \% 5D = \&Instituciones \% 5Bid. estado\%5D=\&Instituciones\%5Bid_subsis tema\%5D=\&Instituciones\%5Bcve_campo_amplio $\% 5 \mathrm{D}=\& y t 0=$ Buscar

United Nations Development Programme. (2001). Human Development Report 2001. Oxford, United Kingdom: Oxford University Press.

World Bank, (1993). The East Asian miracle. Oxford, United Kingdom: Oxford University Press. Retrieved from http:// documents.worldbank.org/curated/ en/975081468244550798/pdf/multipage.pdf

World Bank, (2003). World development report 2003. Oxford, United Kingdom: Oxford University Press. Retrieved from https:// openknowledge.worldbank.org/handle/10986/5985 